\title{
Convergence and stability of line search methods for unconstrained optimization.
}

\begin{abstract}
This paper explores the stability of general line search methods in the sense of Lyapunov, for minimizing a smooth nonlinear function. In particular we give sufficient conditions for a line search method to be globally asymptotical stable. Our analysis suggests that the proposed sufficient conditions for asymptotical stability is equivalent to the Zoutendijk-type conditions in conventional global convergence analysis.
\end{abstract}

Keyword: Global convergence; Globally asymptotical stability; Line search methods; Lyapunov stability; Unconstrained optimization. 